\title{
Procedures for Homogeneous Anionic Polymerization
}

\author{
Lewis J. Fetters* \\ Institute for Materials Research, National Bureau of Standards, Washington, D.C. 20234
}

(May 11, 1966)

\begin{abstract}
This report is a review of the procedures and equipment used in the preparation of polymers of predictable molecular weights and narrow molecular weight distributions. The monomers considered in detail are styrene, $\alpha$-methylstyrene, isoprene, and butadiene.
\end{abstract}

Key Words: Homogeneous anionic polymerization, monodisperse, polystyrene, poly- $\alpha$-methylstyrene, polyisoprene, and polybutadiene.

\section{Introduction}

Organometallic actuated addition polymerizations of unsaturated compounds (vinyl, diene, etc.) in hydrocarbon and ether media have received considerable attention. Many of these polymerizations are unusual in that spontaneous termination is avoidable through the judicious choice of experimental conditions; a feature first fully appreciated by Szwarc $[1,2]{ }^{1}$ Of considerable experimental value is the fact that the deactivation of these "living" polymercarbanions may be accomplished at the leisure of the experimenter by the selective addition of a reagent suited to meet a particular need. This retention of chain-end activity is a feature that facilitates investigations of the thermodynamics of polymerization processes, simplifies studies of polymerization kinetics, and permits synthesis of novel macromolecules.

These systems can therefore consist of active chains which will continue to incorporate monomer until monomer depletion is accomplished. Hence, if each center of propagation is afforded equal opportunity of growth, the resulting product will possess a homogeneous molecular weight [3]. As a result, many studies of homogeneous anionic polymerization have had as a foremost goal the synthesis of polymers of predictable molecular weight and predeterminable molecular weight distributions. Obviously, this goal can only be attained in the absence of any constituent capable of reacting with these carbanions, e.g., acids, alcohols, or the usual atmospheric components.

The general case of a termination-free system received treatment by Flory [3], who showed that the growth of a fixed number of active chains leads to molecular weight distributions approaching monodispersity, i.e., the Poisson distribution. These

* National Academy of Sciences-National Research Council Postdoctoral Resident Research Associate.

'Figures in brackets indicate the literature references at the end of this paper. molecular weight distributions may be represented by the Poisson function,

$$
\begin{gathered}
W\left(K, \bar{X}_{n_{0}}\right)=\left[K\left(\bar{X}_{n_{0}}-1\right)^{K-1} \exp \left(1-\bar{X}_{n_{0}}\right)\right] /\left[\bar{X}_{n_{0}}(K-1) !\right] \\
K=1,2,3,4, \ldots
\end{gathered}
$$

where $W\left(K, \bar{X}_{n_{0}}\right)$ denotes the weight fraction of $K$-mers residing in a mixture of chains of number average chain length $X_{n_{0}}$. For these systems, the weight-tonumber average chain length ratio is given as:

$$
\bar{X}_{w_{0}} / \bar{X}_{n_{0}}=1+1 / \bar{X}_{n_{0}}-1 / \bar{X}_{n_{0}}^{2} .
$$

Such distributions have not been achieved, or even approached, until quite recently with monomers such as styrene [4-15], $\alpha$-methylstyrene [16, 17], isoprene [18-21], and butadiene [21-24]. This was due principally to various factors which combined to veil the true possibilities of these systems. These factors included the presence of impurities or solvents which could terminate or transfer with the growing chains, a slow initiation reaction, a depropagation step (equilibrium polymerization) or the presence of interchange reactions between polymer chains. The latter two phenomena are encountered in the polymerization of cyclic siloxanes [25, 26].

Hence, the fulfillment of the following conditions is necessary if precise control over molecular weight and molecular weight distribution is to be achieved: (a) rigorous exclusion of substances capable of causing termination or transfer; (b) uniformity of reaction conditions with regard to temperature and concentration of reactants; (c) a high ratio of the apparent initiation to propagation rates; and (d) the absence of depropagation steps and bond interchange reactions. In view of increasing interest in the study of bulk and solution properties of polymers with narrow distributions of molecular weights, as well as those of precisely constructed copolymers and "star" macro- 
molecules, the purpose of this communication is to review the techniques by which the preparation of these polymers may be achieved. Results from various laboratories have indicated that the high-vacuum technique provides the most reliable method for the successful attainment of these goals. As will be discussed later, among those who have realized the potential of these techniques have been Bywater and Worsfold, Wenger, Szwarc, Rempp, and Morton.

The monomers considered herein are styrene, $\alpha$ methylstyrene, isoprene, and butadiene. The polymerization initiators described are the isomers of butyllithium. These monofunctional initiators provide several advantages over the electron transfer initiators, e.g., sodium biphenyl or sodium naphthalene. Addition of an electron transfer initiator to an ether solution of monomer results in the formation of an ion-radical $[1,2]$ which eventually undergoes a combination reaction with the resultant generation of a polymeric species possessing a carbanion at each end. In preparing the difunctional species, the accidental deactivation of a fraction of the carbanions will result in a bimodal molecular weight distribution $[5,7,16]$, i.e., the material will possess a portion that will have onehalf the molecular weight of the remaining polymer.

For the case of the polymerization of styrene in an ether such as tetrahydrofuran (THF), complications arise as a consequence of the rapidity of the propagation reaction $[27,28]$. In order to maintain homogeneous reaction conditions, it is necessary to add monomer very slowly, either by distillation or dropwise addition, to a rapidly agitated solution. The presence of trace impurities in the monomer will cause a broadening of the molecular weight distribution because of continuous termination of chain ends throughout the duration of the polymerization. This assumes a constant rate of replenishment of any impurity. Szwarc has shown [29] that when an anion deactivator is present in the monomer at concentrations sufficient to annihilate the entire initial polymercarbanion population by the time the monomer supply is exhausted, the resultant sample will possess the "most probable" molecular weight distribution.

The monofunctional organolithium-initiated polymerization of styrene and other monomers in hydrocarbon media offers several distinct advantages over reactions carried out in tetrahydrofuran. The slow propagation rates [30] permit the addition of the monomer to the solvent-initiator solution in a batchwise fashion. Hence, any impurities will react on mixing with the initiator. If a fraction of the total initiator is destroyed, the molecular weight distribution will not be broadened, although an increase in the molecular weight will occur. The effect on the distribution of a slow termination reaction, comparable in speed to the comsumption of monomer, has received consideration by Coleman et al., [31]. Fortunately, for certain monomers the occurrence of such reactions is avoidable through the proper selection of solvent and by the application of the procedures to be outlined shortly.

\section{Experimental Techniques}

\subsection{High Vacuum Apparatus}

A high vacuum apparatus, figure 1 , permits the attainment of the rigorous experimental conditions necessary to prevent the termination of a reaction by the presence of contaminants. Therefore, all monomer and solvent purifications and polymerizations are carried out on the vacuum line, or in sealed evacuated vessels. The vacuum apparatus is composed of an oil pump (1) working in concert with a mercury diffusion pump (2). In a leak-free system pressures in the range of $10^{-5}$ to $10^{-6} \mathrm{~mm} \mathrm{Hg}$ are obtainable. A liquid nitrogen trap (3) is used to condense any condensable gases so that the pressure registered on the McLeod gage (4) is exclusively that of noncondensable species. The nitrogen trap also prevents the passing of mercury vapor from the diffusion pump into the main manifold (5).

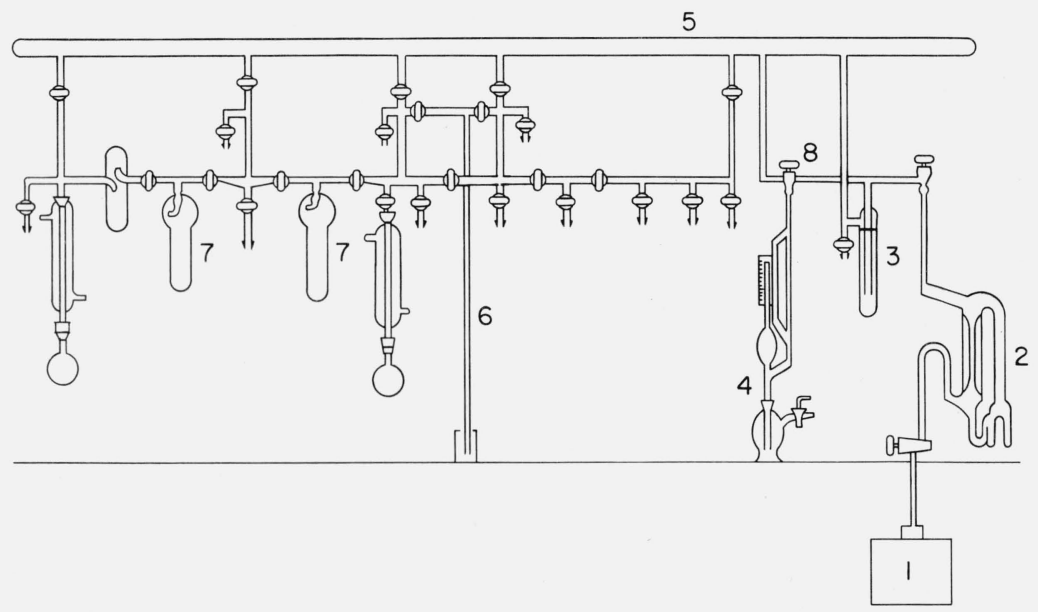

FigURE 1. High vacuum apparatus. 
The pressure release (6) is a tube over $76 \mathrm{~cm}$ in length with one end connected to the vacuum appara" tus and the other end submerged in a mercury pool. When substances with low boiling points, e.g., butadiene, ethylene oxide, etc., are used in vacuum systems there exists the possibility that pressure may build up to the point of fracturing the apparatus or blowing temporarily attached pieces of equipment off the line. The purpose of the pressure release is to relieve such pressure in the apparatus and still maintain an airtight system. Furthermore, the fall of the mercury column provides a visual indication of the onset of such a pressure increase.

\section{a. The Main Manifold}

A manifold of this type consists of a 4 to $6 \mathrm{~cm}$ diameter section of Pyrex glass tubing, clamped in place on a metal supporting frame. The vacuum stopcocks connecting the main manifold to the secondary one possess stopper bores of 6 to $10 \mathrm{~mm}$. The stopcocks placed in the secondary manifold are those with $4 \mathrm{~mm}$ stopper bores. The connecting tubes of the secondary manifold are made of $12 \mathrm{~mm}$ diam heavy wall Pyrex tubing, since this is about the largest size of glass tubing that is practical in constructing a large and rigid piece of apparatus where proper annealing is impractical. Apiezon $N$ hydrocarbon grease is recommended for use in the main manifold whereas silicon grease should be used in the secondary manifold stopcocks since this lubricant is highly resistant to the passage of ether and hydrocarbon vapors. After the application of grease to a stopcock, the system should be pumped for several hours in order to remove trace amounts of moisture from the freshly applied grease.

Graduated cylinders (7) are used to measure out approximate amounts of solvent or monomer prior to final collection. These pieces of equipment may either be permanently attached to the line or equipped with ground glass joints for temporary placement.

The entrance stopcock (8) of the McLeod gage to the vacuum line is located so as to register pressure on either side of the nitrogen trap. During the early stages of evacuation readings taken between the cold trap and the manifold will indicate a lower pressure than will measurements made directly from the manifold. This disparity is due to the removal of condensable gas in the nitrogen trap. Hence the readings referred to herein are those registered directly from the main manifold.

\subsection{General High Vacuum Techniques and Equipment}

All reactors and ampoules are constructed from Pyrex glassware. Prior to assembly of the equipment, the glassware should be cleaned with either cleaning solution or hydrofluoric acid, rinsed with a dilute solution of sodium hydroxide, washed with distilled water, and dried.
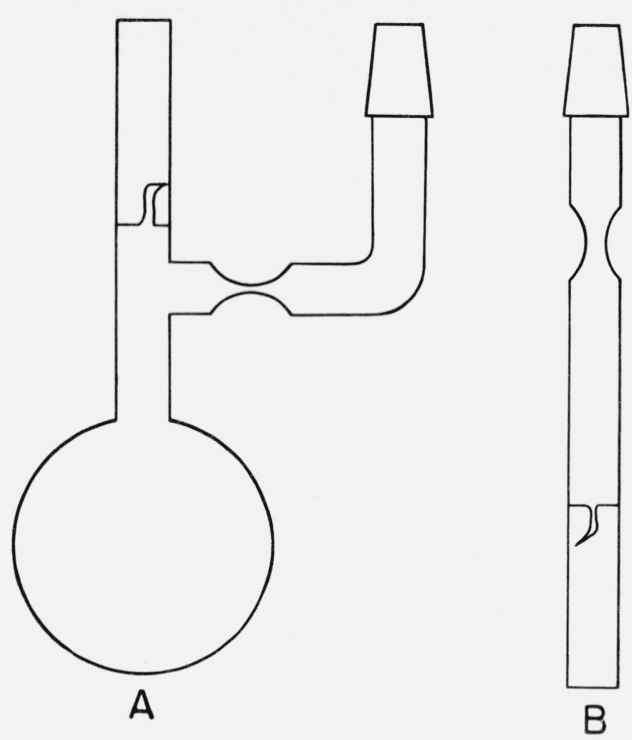

FIGURE 2. Monomer-solvent ampoules.

Figure 2 depicts typical monomer-solvent ampoules. The breakseals are usually those made from $12 \mathrm{~mm}$ diam tubing. A convenient size of ground glass joint is the $14 / 35$ type.

After construction of the equipment, the glassware is evacuated on the vacuum line and tested with a Tesla coil for leaks. When it is ascertained that the apparatus is structurally sound, the glassware is heated, with a handtorch adjusted to give a soft blue flame, until the yellow sodium flame is given off from the glass surface. The glassware during this heating period is continuously being evacuated. Intermittent heating is continued until a pressure of $\sim 10^{-6} \mathrm{~mm} \mathrm{Hg}$ is recorded on the McLeod gage. The purpose of this treatment is to remove the adsorbed gas and water film which are present on the surface of the glassware.

Rupturing the breakseals is accomplished with the aid of an iron nail enclosed in a glass envelope. The glass jacket is made from tubing of the appropriate diameter rounded off at one end. The nail is put in place with either glass wool or asbestos fibers to pack it solidly in place. A constriction is then placed above the nail and the tube sealed onto a manifold for eventual evacuation. After thorough evacuation is accomplished, the nail is sealed off and the end of the envelope rounded off.

\section{a. Distillation and Degassing Procedures for Liquids in a Vacuum System}

In the absence of appreciable amounts of noncondensable gases, transfer of volatile materials to a cooled zone is rapidly effected. However, in the 
presence of even small amounts of noncondensable gases, the diffusion of condensable materials is impeded. It is therefore necessary to maintain the pressure below $\sim 10^{-3} \mathrm{~mm} \mathrm{Hg}$ in order to carry out transfer of condensable materials at a practical rate. Thus, it is quite important to exhaustively degas all liquids prior to any subsequent operation, such as distillation, drying, and collection.

The removal of air is carried out as follows. The flask containing the liquid to be degassed is connected to the vacuum line and cooled until frozen. An appropriate cooling bath such as liquid nitrogen or a dry-icealcohol mixture is used, depending on the freezing point of the liquid being degassed.

When the substance is frozen, the flask is evacuated by opening the stopcock between it and the vacuum line. After approximately $5 \mathrm{~min}$, the flask is isolated from the vacuum line and the frozen material warmed to room temperature. To expedite melting, the flask may be submerged in an alcohol bath. The freezing and thawing cycle is repeated until the pressure registered on the McLeod gage is of the order of $10^{-5} \mathrm{~mm}$ $\mathrm{Hg}$ with the material in the frozen state. The material is then considered degassed and is ready for subsequent treatment. A supplementary discussion on the distillation and collection of liquid hydrocarbons can be found elsewhere [32].

\section{b. Preparation of Sodium Mirrors}

For use as a drying agent for trace quantities of moisture, a thin sodium mirror is advantageous. This mode of drying is not to be confused with the usual ritual of drying organic liquids with large lumps of sodium, which in the usual course of events all too soon accumulate a coating of oxide and hydroxide. These mirrors are readily prepared on a high vacuum line as follows. Approximately $1 / 8$ to $1 / 4 \mathrm{~g}$ of dry sodium metal (not kept under mineral oil) is placed in a flask, which may be of any size up to 1 to 2 liters capacity, and connected to the vacuum line. The flask is evacuated until a pressure of $10^{-4} \mathrm{~mm} \mathrm{Hg}$ or less is obtained.

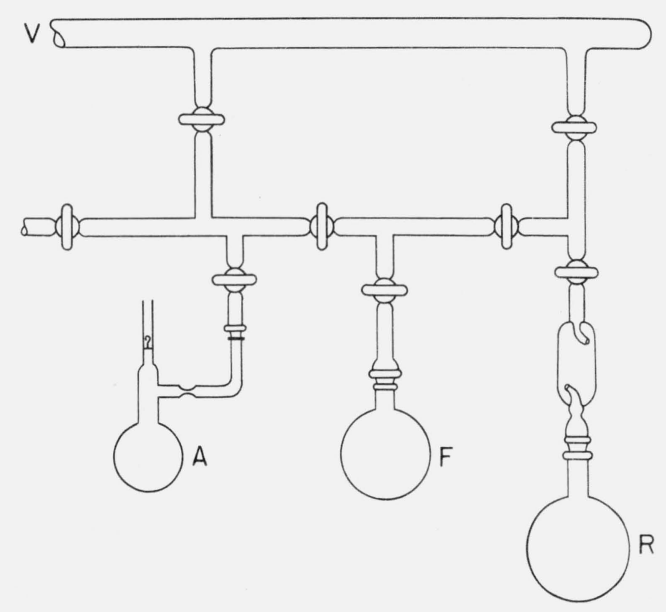

FIGURE 3. Solvent purification apparatus.
While still being evacuated, a small area at the bottom of the flask is gently and intermittently heated with a small, soft flame from a hand torch until the metal begins to melt. The metal will then vaporize to form the mirror. Excess heating may cause the flask to crack. Once the mirror is formed, evacuation is continued until the flask has cooled to room temperature. Then the liquid to be dried is distilled onto the sodium film. Once the liquid is in contact with the mirror, it should receive further degassing in order to remove any hydrogen formed by the reaction of moisture with sodium. Exposure to two or three mirrors is usually sufficient to ensure dryness.

\section{c. Preparation of Sodium-Potassium Alloy}

The alloy of sodium and potassium is prepared in a flask $(\mathrm{R})$ as noted in figure 3. Approximately equal parts by weight of the two alkali metals are used in the preparation. The use of this ratio ensures the existence of the alloy in the liquid state at room temperature. In this form it serves as a very efficient drying agent for hydrocarbon solvents. The total weight of the two metals need not exceed $1 \mathrm{~g}$.

The sodium used in this preparation should be as free as possible from hydroxide. The mineral oil is removed from the potassium by rinsing the metal in dry benzene or toluene. The rinsed lumps must be covered by solvent at all times during handling and transfer.

Following the placing of the flask containing the two alkali metals, the covering solvent and a stirring bar on the vacuum line, the apparatus is then carefully evacuated and the covering solvent distilled off and allowed to collect in a second flask cooled by a dry-ice-alcohol mixture. After the attainment of a good vacuum $\left(\sim 10^{-5} \mathrm{~mm} \mathrm{Hg}\right)$, the flask is gently heated with a soft flame from the gas torch until the liquid alloy is formed. The glass-enclosed stirring bar is held against the side of the flask until the alloy cools to room temperature. Extreme care must be exercised during the heating operation since a crack in the flask would result in spontaneous ignition of the alloy.

A consequence of this characteristic is that a flask containing the alloy should never be allowed to lose its vacuum unless the alloy is covered with solvent. In this condition, the flask can be removed from the vacuum line for the decomposition of the residual alloy. The flask should be placed in a hood and the alloy destroyed by the addition of cold (dry-ice temperature) isopropyl alcohol. The reaction must be monitored until the decomposition is complete.

\section{d. Preparation of Organolithium Purge Ampoule}

The final step in the purification of hydrocarbon solvents and of diene and vinyl monomers is exposure to an organolithium. Butyllithium is used except for styrene. Fluorenyllithium is used for this mono- 
mer. A flask is constructed with a sidearm constriction, to which is added an ampoule of organolithium. The apparatus is placed on the vacuum line and evacuated. The solution of organolithium is added to the flask by rupturing the breakseal. The solvent is then distilled away. Exposure of either solvent or monomer to the organolithium ensures eradication of the final vestige of any polymerization terminating impurity.

\section{Preparation of Materials}

From a practical standpoint it is necessary to delineate what is meant by pure materials. We are concerned with the removal from the various reaction components of any material capable either of carbanion deactivation or of influencing the reaction pattern of the polymerization. The exclusion of this latter class of impurities, e.g., polar compounds, is of importance in the polymerization of dienes since the intrusion of even trace amounts of certain polar species can alter the microstructure of the polydienes.

\subsection{Solvent Purification}

\section{a. Tetrahydrofuran}

This ether is stored over finely ground calcium hydride and stirred for several days on the vacuum line (fig. 3). After degassing, the ether is distilled, with the aid of a dry-ice-alcohol bath, over a succession of sodium mirrors $(\mathrm{F})$ until the ether produces no visible degradation of the mirror at room temperature. The ether is again degassed and subsequently distilled into a flask (R), usually of one liter capacity, containing approximately one-quarter gram of biphenyl or naphthalene and one-half to one gram of sodium or potassium. The addition of the ether to the alkali metal and the organic aromatic species soon results in the formation of a dark blue (biphenyl) or dark green (naphthalene) solution. The excess alkali metal is necessary to ensure complete conversion of the aromatic to the complex since any unreacted aromatic will sublime in subsequent distillations of the ether.

When naphthalene is used, a dark red color may eventually develop in the solution. This is due to the reaction between the alkali metal naphthalene and an isomer of dihydronaphthalene [33]. Either 1,2- or 1,4-dihydronaphthalene may be generated by the reaction of water with the alkali metal naphthalene complex. The 1,2-isomer will polymerize to give an insoluble low molecular weight product while the 1,4-form will react with the alkali metal naphthalene but will not polymerize [34].

Measured quantities of ether can be flash-distilled as needed into an appropriately evacuated, calibrated, and weighed ampoule (A). The collection ampoule may be given a thin sodium film in order to ensure dryness. Shielding the collected ether from any ultraviolet radiant-energy source, including sunlight, should be practiced as a precaution against peroxide formation and other deleterious side reactions. This can best be accomplished by storing the ampoules in the refrigerator. Other ethers (dioxane, methyltetrahydrofuran, ethyl ether, etc.) can be processed by this procedure.

\section{b. Benzene}

This solvent is stored over sulfuric acid, with stirring, for at least one week. The material is then placed on the vacuum line (fig. 3) and degassed. The liquid is then distilled to flask (R) which contains the alloy of sodium and potassium. Stirring is then commenced and continued for $24 \mathrm{hr}$. After degassing, portions are distilled to a sodium mirror $(\mathrm{F})$. From flask $(\mathrm{F})$, the benzene can then be distilled to a flask containing butyllithium. The residence time of the benzene with the butyllithium is usually of several hours duration. Then measured quantities are collected in ampoule (A), as needed, or distilled directly into a reactor.

\section{c. Tolvene}

Toluene is processed in a fashion similar to that used for benzene. The only difference is the deletion of the exposure to sulfuric acid. In its stead, the solvent is exposed to lithium alluminum hydride prior to treatment on the alkali metal alloy.

\section{d. $n$-Hexane}

The purification process for $n$-hexane is identical to that of benzene.

\subsection{Monomer Purification}

\section{a. Styrene}

Styrene is purified in a vacuum apparatus as follows. A flask (A), figure 4, is prepared containing a slurry of finely ground calcium hydride in styrene. The mixture is then throroughly pumped and degassed.

The contents $\left(\sim 100\right.$ to $\left.150 \mathrm{~cm}^{3}\right)$ are then agitated by a magnetic stirrer for several days with periodic degassing. During this period, the viscosity of the mixture will increase slightly, indicative of the formation of a small amount of low molecular weight polymer. At the end of this treatment the styrene is distilled through the manifold into a chain flask where the monomer is circulated through a sodium coated bulk (B) and condensed in flask (D) by a dry-iceisopropanol mixture. The sodium-coated bulb is formed by placing approximately $0.1 \mathrm{~g}$ of sodium into the side arm of the flask and gently melting the alkali metal. The metal vapor will then condense on the upper portion of the chain flask. This mode of monomer purification was developed by Hall [8] and Meier [22]. 


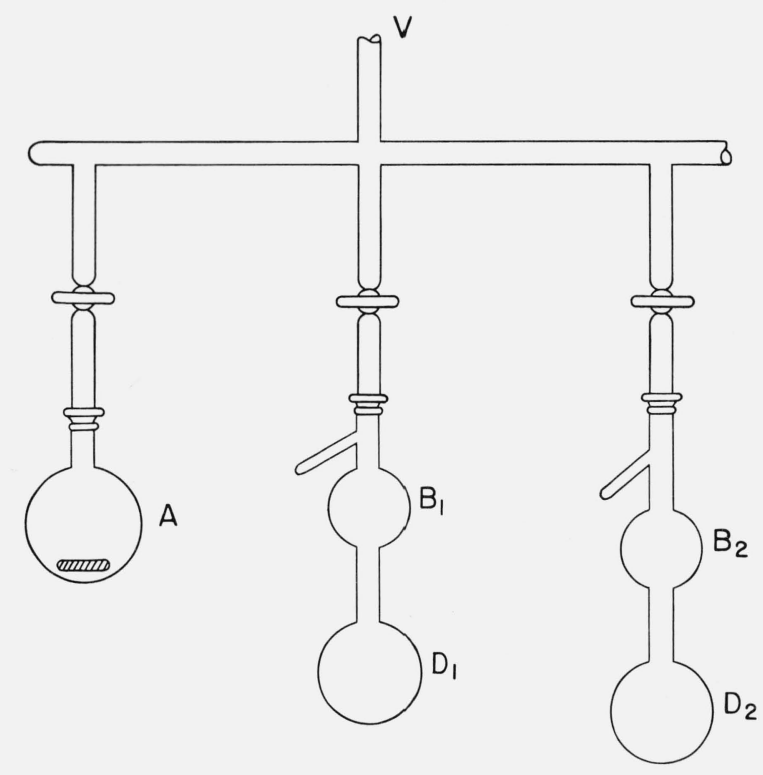

Figure 4. Apparatus for the purification of styrene.

The monomer is then distilled into several additional flasks prior to its final collection. Exposure to a minimum of three flasks is recommended. This procedure has proved to be adequate for reducing contaminants to low levels. However, if desired, the monomer may also be exposed to fluorenyllithium prior to final collection. Unlike butyllithium, this organolithium will not react with styrene [35]. Care must be exercised in the final collection step to ensure that residual fluorene does not sublime into the collection ampoule. This can be circumvented by distilling the monomer into a chain flask prior to the final collection.

\section{b. $\alpha$-Methylstyrene}

This monomer can be purified by procedures similar to those used for styrene. Polymer formation during purification is negligible due to the low ceiling tem-

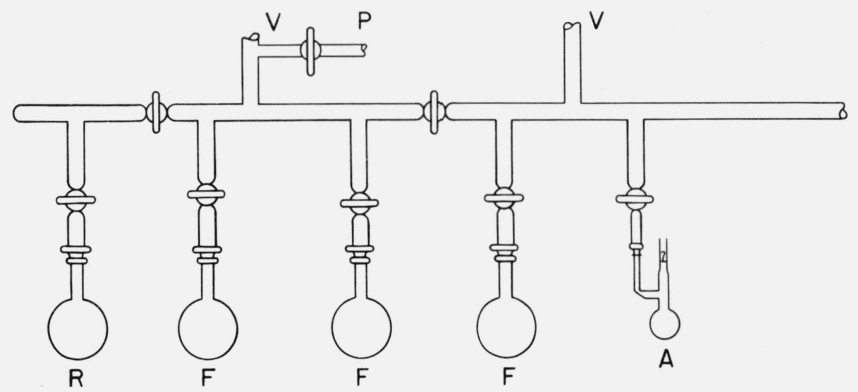

FigURE 5. Apparatus for the purification of diene monomers. perature of the monomer. Several separate exposures are sufficient to dry the monomer rigorously. After purification the monomer is collected and stored in a manner identical to styrene.

\section{c. Isoprene}

This diene lends itself to ready purification with the use of a high vacuum manifold as depicted in figure 5 . The monomer is exposed in flask $(\mathrm{R})$ to calcium hydride and stirred for $24 \mathrm{hr}$. The monomer is then degassed, with nitrogen used to freeze the monomer. The monomer is flash-distilled three times to flasks $(\mathbf{F})$ which are coated with freshly sublimed sodium. The first distillation removes, as it does with the other monomers, any inhibitor. The monomer is then exposed to butyllithium at room temperature for $15 \mathrm{~min}$ or so. Finally, designated quantities of monomer are collected in ampoules (A), where final degassing is accomplished. The ampoules are sealed off, weighed, and stored at $\sim 0{ }^{\circ} \mathrm{C}$ until used.

\section{d. Butadiene}

The section of the manifold used is fitted with a mercury column pressure release $(\mathrm{P})$, as depicted in figure 5. The drying procedure is identical to that of isoprene. Due to the low boiling point $\left(-4.4{ }^{\circ} \mathrm{C}\right)$ of butadiene, it is necessary to maintain the temperature at a low level with a dry-ice-alcohol bath.

\subsection{Preparation of Organolithiums}

$$
\text { a. n-Butyllithium }
$$

The apparatus used to prepare $n$-butyllithium is illustrated in figure 6 . An amount of di- $n$-butylmercury sufficient to make up a one molar solution of organolithium is placed in a clean dry flask fitted with a standard-taper joint vacuum connection and a breakable seal. After the flask is degassed, dry $n$ hexane $(50-75 \mathrm{ml})$ is distilled in, the contents are again degassed, and the flask is sealed off. This ampoule $(\mathrm{S})$ is then sealed to the apparatus $(\mathrm{R})$ as illustrated in figure 6 . Lithium ribbon or wire with

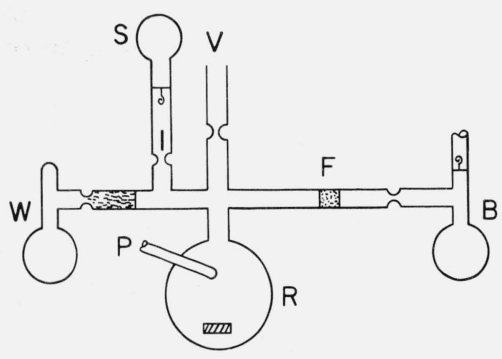

FIGURE 6. Apparatus for the preparation of n-butyllithium. 
the petroleum coating intact is charged into $(\mathrm{R})$, a reaction flask of about $300 \mathrm{~cm}^{3}$ capacity, through $(\mathrm{P})$ an open side arm; $(\mathrm{P})$ is then sealed off. After pumping to high vacuum, $50 \mathrm{~cm}^{3}$ or so of anhydrous hexane is distilled into (R) and degassed. After degassing, the apparatus is removed from the vacuum line and the contents of the flask $(R)$ are stirred for several hours. The resultant solution of petrolatum in hexane is then decanted through a glass wool plug to (W). Clean hexane is then distilled back to $(\mathrm{R})$ and the cycle repeated until the lithium surface is cleaned and all soluble residue removed from flask $(\mathrm{R})$. Flask $(\mathrm{W})$ is then removed by sealing off. The di- $n$-butylmercury-hexane mixture is then added to the lithium in (R) and stirring commenced at room temperature. At the end of the reaction period, usually of several week's duration, the $n$-butyllithium-hexane solution is transferred to (B) through a medium porosity fritted glass disk $(\mathrm{F})$. (R) is rinsed several times by back-distillation in order to ensure a clean transfer. The content of $(B)$ is divided into several ampoules having breakable seals. Analysis is made according to the method of Gilman $[36,37]$.

\section{b. Isomers of Butyllithium}

A vacuum modification of the method reported by Telalaeva and Kocheskov [38] is used for the preparation of the remaining isomers of butyllithium. The appropriate organo-chloride or bromide (sec-butyl, iso-butyl, or $t$-butyl) is placed under vacuum in a manner similar to that of the di- $n$-butylmercury. Reaction progress is accompanied by the formation of the lithium halide. After completion, the slurry is filtered and the solution collected in flask (B). This procedure has also been used by Hall [8] for the preparation of ethyllithium.

\section{c. Fluorenyllithium}

The preparation of fluorenyllithium for monomer purification is a modification of procedures presented elsewhere [39]. A reaction ampoule is constructed to which is attached a breakseal and a sidearm constriction. Attached to the sidearm constriction is an ampoule of $n$-butyllithium. The amount of fluorene added is always in slight excess $(\sim 5$ mole $\%)$ of the added $n$-butyllithium. After thorough evacuation, about $75 \mathrm{~cm}^{3}$ of dry benzene is distilled into the reactor and the reaction allowed to proceed until completion. Complete conversion of the $n$-butyllithium must be accomplished since any residual organolithium will react with styrene. After completion of the reaction, the fluorenyllithium is divided into ampoules for use.

\section{d. Polyorganolithium "Seed" Preparation}

It is now recognized [30, 40-48] that the reaction between organolithiums and unsaturated monomers is a relatively sluggish process in hydrocarbon media.
It should be remembered that initiation is a one-step reaction whereas the growth of a chain involves a large number of consecutive addition steps. Hence, even if the individual initiation is somewhat slower than the individual propagation reaction, the actual formation of a chain may take considerably longer than the time required for complete initiation. However, if the initiation reaction is two or three orders of magnitude slower than propagation, then the two processes may become competitive.

The slowness of the initiation reaction results in a process in which the kinetics of initiation and propagation become rather complex [41-46]. The deciphering of the order of the initiation reaction in organolithium for these polymerizations has thus far proved to be highly troublesome. From a synthesis viewpoint, the simultaneous occurrence of the two reaction steps lead to relatively broad molecular weight distributions $\left(\bar{M}_{w} / \bar{M}_{n}\right.$ 1.3-1.4) [49, 50]. This complex behavior results from, in part, the ability of the initiating organolithium to form associated species with itself [51] as well as with polymer-lithium [52]. Kinetic [30, 40-48, $53-55]$ and molecular weight measurements [47, 52-57] indicate that the various organolithium species participating in initiation and propagation form diverse associated species. The unambiguous assignment of the kinetic orders of the various processes and the elucidation of the association states of the various species and the nature of the reactive species, remain incomplete. $^{2}$

The effect of slow initiation can be partially circumvented by using a two-stage polymerization method, i.e., a "seeding" technique. In this procedure, a small quantity of monomer and solvent are mixed in an ampoule with an appropriate amount of the initiating organolithium. The low molecular weight polyorganolithium is formed under conditions that are not practicable or convenient for the preparation of the high molecular weight polymer.

For the preparation of low molecular weight polystyryllithium, styrene (in benzene) is mixed with $n$-butyllithium and the reaction permitted to proceed at approximately $30{ }^{\circ} \mathrm{C}$ for $12 \mathrm{hr}$ or so. It has been determined [30] that for complete conversion of $n$-butyllithium to polystyryllithium, the following must hold,

$$
[M]_{0}[I]_{0}^{-4 / 3}>504
$$

where $[M]_{0}$ and $[I]_{0}$ are the initial monomer and organolithium concentrations, respectively. At the completion of the initiation reaction the value of $\bar{M}_{n}$, the number-average molecular weight, is

$$
\bar{M}_{n}=\frac{\text { grams of monomer }}{\text { moles of initiator }} \text {. }
$$

${ }^{2}$ For an interesting probe into this problem see T. L. Brown, J. Organometallic Chem. 5, 191 (1966); Advance in Organometallic Chem. 3, Ed. by F. G. A. Stone and R. West, p. 365, Academic Press, New York, 1965. 
This relation is valid since each of the monofunctional initiating organolithium molecules generates one polymer chain. The relatively broad distribution of the "seed" polystyryllithium is overshadowed by the great excess of additional polymerization where all growing chains have equal probability of growth.

It should be mentioned that polystyryllithium in benzene undergoes a slow isomerization reaction at elevated $\left(\sim 75^{\circ} \mathrm{C}\right)$ temperatures [58]. This reaction was first noted by Szware [59] and co-workers for polystyrylsodium in tetrahydrofuran. Thus the "seed" preparation, as well as the polymerization reaction, should be accomplished at relatively mild temperatures.

The preparation of the polyisoprenyllithium "seed" can be carried out in a manner similar to that used for polystyryllithium. For this procedure $n$-hexane is used as the solvent. Complete conversion of $n$-butyllithium to polyisoprenyllithium can be accomplished as follows [42]. A solution of $n$-hexane and isoprene is made with an isoprene concentration of $\sim 8$ moles liter $^{-1}$. This is then mixed with an appropriate amount of $n$-butyllithium to yield a polymer with a number average molecular weight of $\sim 10,000$. The reaction is carried out at a temperature of $0{ }^{\circ} \mathrm{C}$. This relatively mild temperature is used since the apparent energy of activation for initiation $(\sim 18 \mathrm{~kg}$ cal $)$ has been found to be lower than for propagation $(\sim 22 \mathrm{~kg}$ cal $)$ $[40,42]$. These values lack their usual physical significance since they contain contributions from the heats of dissociation of the alkyllithium, polyisoprenyllithium, and the cross-associated species. Therefore, the initiation efficiency is found to increase at lower temperatures [42]. This is convenient from an experimental point of view, since it permits working at high monomer and initiator levels without fear of the exotherm becoming uncontrollable.

These conditions may also be applied for the formation of polybutadienyllithium. Evidence exists $[40,41]$ that the rate of initiation of butadiene is faster than that of isoprene under identical conditions. This, coupled with the fact that the apparent rate of propagation for butadiene is less than that of isoprene, greatly enhances the quantitative conversion of the $n$-butyllithium to polybutadienyllithium under the conditions outlined above.

It is of interest to record that the preparation of these low molecular weight polymeric-lithiums free from unreacted initiating organolithium, is facilitated by the substitution of sec-, iso-, or $t$-butyllithium for $n$-butyllithium. Kuntz [46] and Hsieh [41] have noted that these isomers are more efficient in initiating isoprene and butadiene than is $n$-butyllithium. Although no truly quantitative study of the relative apparent rates of initiation of these various isomers of butyllithium is currently available, the indication does exist that under the conditions outlined herein, replacement of $n$-butyllithium with one of the other isomers will result in a faster and more efficient "seed" preparation. The enhanced initiation rates appear to

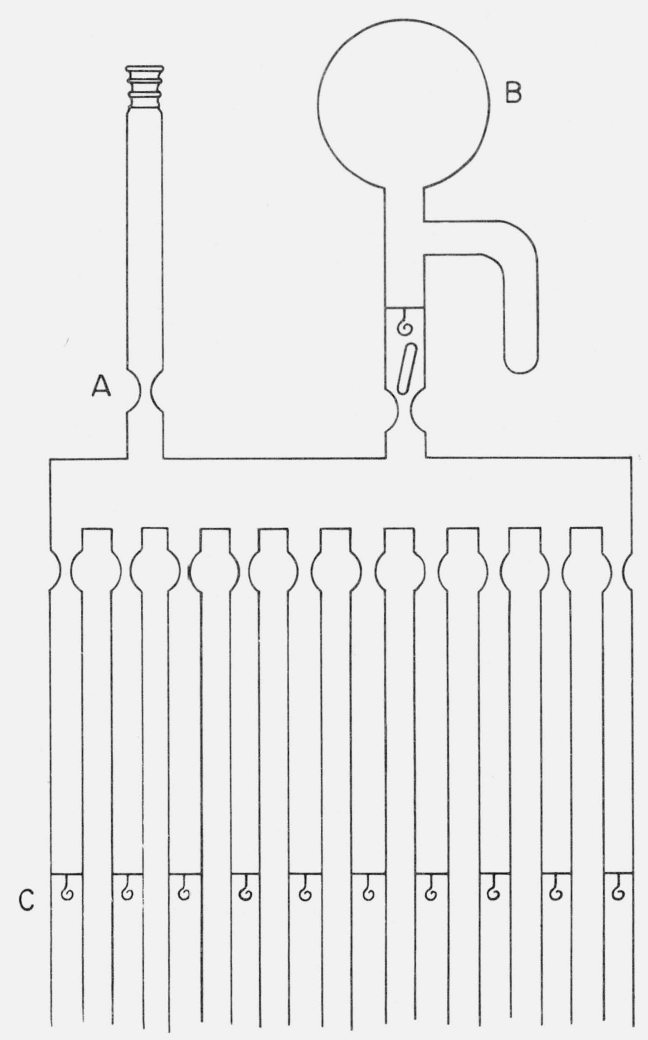

Figure 7. Apparatus for subdivision of initiator solution.

be due, in part, to the lower degrees of self-association exhibited by these isomers of butyllithium [60].

After completion of the "seed" preparation, the flask (B) containing the solution is sealed onto a manifold containing approximately 10 ampoules, as shown in figure 7 . The manifold is then connected to the high-vacuum apparatus, evacuated, leak-tested, and flamed. After removal from the high-vacuum line by sealing the manifold off at the constriction (A), the breakseal of the "seed" solution is ruptured thus permitting the mixture to flow into the breakseal ampoules. Flask (B) is removed by sealing it off at the constriction which has been rinsed by refluxing solvent. The manifold is then inverted so that a uniform solution in all of the ampoules is achieved. The manifold is then allowed to stand for approximately $24 \mathrm{hr}$ in order that each constriction has sufficient time to drain free of residual polymer. The refluxing of solvent onto the constrictions is not recommended since distillation of solvent among the ampoules will cause fluctuations in the lithium concentration. The same procedure is used for the dilution of the initiatıng organolithiums.

The determination of the lithium content is carried out as follows. The volume of solution in the $12 \mathrm{~mm}$ diamater sealed breakseal tubes is estimated by a comparison with a similar tube which is calibrated in 1 $\mathrm{ml}$ increments. A more accurate measure can be obtained, after use of the solution, by titration of the used 
ampoule by means of a $10 \mathrm{ml}$ micro-buret. After the volume of the sample is determined, the decomposed organolithium is titrated with either $0.01 \mathrm{~N}$ or $0.1 \mathrm{~N}$ hydrochloric acid to a phenolphthalein end point. For the polymeric-lithium, the titration is carried out in a neutralized solution (1/1 by volume) of methanol and water. This prevents the coagulated polymer from absorbing either indicator or lithium hydroxide. Two ampoules from each batch are titrated in this manner to determine the lithium content per cubic centimeter of solution. It is also recommended that the Gilman double titration procedure $[36,37]$ be employed on occasion to ascertain the concentration of noninitiating lithium compounds in solution.

\section{Polymerization Procedures}

The procedures outlined in the following will apply, in the main, to all of the monomers covered herein. Where applicable, the necessary variations will be listed in the appropriate place. To perform an experiment, the various ampoules are sealed onto a reactor, figure 8 , in such a manner that the separate components can be conveniently added to the reaction mixture while maintaining a closed system. A vacuum line (V) consisting of a standard-taper ground-glass joint at one end and a constriction at the other is also attached to the reactor vessel in a position that will evenly support the weight of the reactor. The vacuum line is the sole connection and, in many instances, the only support holding the reactor to the vacuum system. It is therefore imperative that the weight of the reactor and its components be well balanced. The respective ampoules pictured contain monomer $(\mathrm{M})$, solvent $(\mathrm{S})$, initiator (I), and terminator (T). A glass wool plug, previously washed with $\mathrm{THF}$, is placed above the constriction of ampoule (S) to provide a filter for any sodium particles which might be carried from the mirror on which the solvent is stored. For most cases the terminator may consist of several drops of welldegassed water or methanol. Sampling ampoules (1) and (2) may be added to $(R)$ if desired.

Once the reactor is assembled as shown in figure 8, it is connected to the high-vacuum apparatus by means of the ground-glass joint on the vacuum tube and evacuated. After several minutes, the reactor is tested with a Tesla coil for pinholes or cracks, especially in the area where the component parts are sealed on. If no pinholes are found, or after those that are found are sealed, the reactor is evacuated until a pressure of $10^{-6} \mathrm{~mm} \mathrm{Hg}$ is obtained. All of the glass surfaces, except the ampoules containing the reaction ingredients, are flamed strongly with a yellow-blue flame from a hand torch. The reactor is continuously under evacuation during this procedure. After $1 \mathrm{hr}$ or so the vacuum is again checked, and if a pressure of $10^{-6} \mathrm{~mm}$ $\mathrm{Hg}$ is obtained, the reactor is sealed off from the high-vacuum line at the constriction.

Prior to the addition of the reactants, the reactor is purged, on occasion, by an additional ampoule of dilute butyllithium. This solution is removed in ampoule (1).

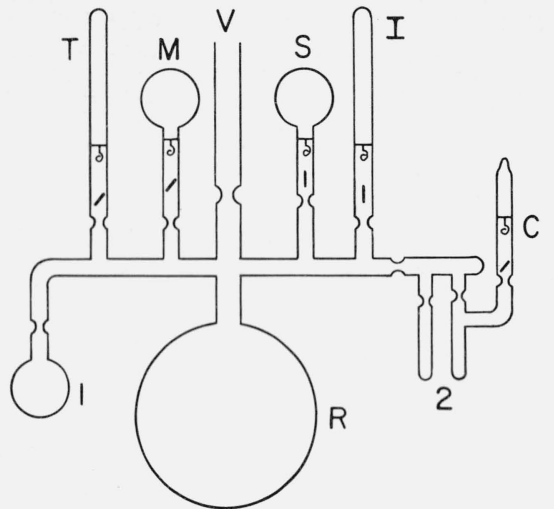

FiguRE 8. Polymerization reactor.

This procedure is used when very large reactors and small initiator charges are employed. However, care must be taken to thoroughly rinse the system free from any traces of the purge catalyst. Fluorenyllithium may also be used as a purge organolithium.

The solvent and initiator are introduced and the initiator ampoule is rinsed by refluxing solvent. The mixture is then allowed to come to the desired reaction temperature and the monomer is added. The monomer generally comprises from 10 to 20 percent by volume, with 5 to $20 \mathrm{~g}$ being a typical charge. All empty ampoules are then removed by sealing off at the respective constrictions. The exact volume of initiator used can then be determined by sealing the ruptured breakseal and titrating to the previously applied mark. If desired, samples may be removed at (2) in order to analyze for the active lithium content of the solution by means of the Gilman double titration. Ampoule (C) contains a THF solution of benzyl chloride, allyl bromide, or 1,2-dibromoethane used in this analysis.

For the styrene polymerization (in benzene or toluene), a procedure may be used which can circumvent the slow initiation reaction. Several workers $[22,61]$ have investigated the effect of small quantities of tetrahydrofuran on the initiation and propagation reactions of styrene in benzene. It was noted that with small concentrations of the ether $([\mathrm{THF}]=[\mathrm{BuLi}])$, the initiation reaction becomes very rapid, i.e., too fast to be measured by the optical methods usually used $[30,61]$ in studies of this system. These characteristics are of value from a synthesis standpoint since it is possible now to prepare samples of monodisperse, low molecular weight $(\sim 1000)$ polystyrene $[14,62]$. The propagation reaction changes its rate in a rather complex fashion with variations in the THF concentration at a constant concentration of butyllithium. These results $[22,61]$ have been tentatively explained on the basis of solvation of the active species 
with the ether. It would appear that the changes in carbanion reactivity are due to the formation of peripherally solvated and solvent-separated ion-pairs. This change in reactivity is a further demonstration of the sensitivity and susceptibility of carbanions to subtle environmental changes.

The use of the ether in the concentration mentioned above eliminates the relatively long period of initiation but does not appreciably alter the rate of the propagation reaction $[22,61]$. This results in an easily controllable polymerization in benzene at moderate $\left(\sim 30^{\circ} \mathrm{C}\right)$ temperatures. Also, this low ratio of ether to organolithium rules out the possibility of any extensive reaction between THF and the organolithium $[8,63,64]$.

In this procedure the measured quantity of ether is added immediately after the addition of monomer. The solution will then turn bright red within several seconds. Only small amounts of ether are required, i.e., $[\mathrm{THF}] /[\mathrm{BuLi}]=1$ to 10 . After thorough mixing, the polymerization is allowed to proceed at the desired temperature $\left(0\right.$ to $\left.30{ }^{\circ} \mathrm{C}\right)$. Depending upon temperature, initiator concentration, and ether concentration, complete conversion can be attained from $15 \mathrm{~min}$ to $8 \mathrm{hr}$.

Atactic polystyrene is prepared [65] under the conditions outlined above. It has been reported [66-68] that polystyrene resulting from organolithiuminitiated polymerizations in nonpolar solvents (toluene) at low $\left(-20\right.$ to $-40{ }^{\circ} \mathrm{C}$ ) temperatures contains a substantial fraction of crystallizable polymer. The presence of any polar material prevents stereoregularity. The crystallizable portion has been identified as isotactic material. It was shown [69] that the formation of the isotactic material was due entirely to the presence of lithium hydroxide, the formation of which was due to the presence of moisture in the reaction mixture. Polystyrene prepared under anhydrous conditions is predominately syndiotactic [69]. Elevation of the reaction temperature above $0{ }^{\circ} \mathrm{C}$ results in the production of atactic material.

In connection with this point, it has been reported [70] that the presence of lithium methoxide in the $n$-butyllithium-initiated polymerization of methyl methacrylate enhances the rate of propagation, increases slightly the amount of isotactic polymer, and facilitates the formation of high molecular weight material. The presence of lithium ethoxide or lithium butoxide increases the rate of initiation of isoprene $[42,47]$. A possible explanation for this is given by Brown [71]. Brown has shown that lithium ethoxide forms a soluble complex with ethyllithium, presumably through the unshared pair of electrons on the oxygen atom. It is therefore possible that these lithium salts can associate or complex with the normal organolithium ion-pair. Their presence may also promote the dissociation of the associated organolithium, as do traces of ethers. In any case, the infiltration of these lithium salts can alter the reactivity of an active organolithium and influence the mode of monomer addition. These findings emphasize the necessity of obtaining a stringently pure system and of maintaining a static reaction environment.
The polymerization of $\alpha$-methylstyrene is carried out in tetrahydrofuran at $-78{ }^{\circ} \mathrm{C}$. This low temperature is necessary since the formation of high polymer is thermodynamically unfavorable at higher temperatures $[72,73]$. Mixing and initiation are carried out at room temperature. At this temperature, initiation will occur without the formation of high polymer. The $n$-butyllithium should be added to the monomer-ether solution in order to retard the reaction [63] between the ether and the organolithium. The reactor is then placed in a dry-ice-alcohol bath and the contents swirled for $10 \mathrm{~min}$ or so while the mixture is cooling. Complete conversion usually occurs in $24 \mathrm{hr}$ [74].

The polymerization of dienes is readily accomplished in hexane at temperatures ranging from 0 to $30{ }^{\circ} \mathrm{C}$. The use of THF to circumvent the "seed" preparation is avoided since the microstructure of these dienes is altered by trace amounts of polar compound [47, $56,57,75]$.

Polyisoprene prepared in pure hydrocarbon media is structurally a close counterpart to Hevea rubber. The cis-1,4 enchainment is enhanced if the organolithium concentration is of the order of $10^{-3}$ to $10^{-4}$ moles liter ${ }^{-1}$ and the reaction temperature remains in the vicinity of $0{ }^{\circ} \mathrm{C}[75]$. Under these conditions, several days are necessary for the attainment of complete conversion. Polybutadiene microstructure appears to be moderately dependent on reaction temperature and initiator concentration [76,77]. Generally a polymer with 10 percent vinyl, 40 percent cis-1,4, and 50 percent trans-1,4 content is obtained.

On termination of the polydienyllithium species, a marked decrease in solution viscosity will occur. This is due to the association which the active chain ends undergo $[30,47,53-57]$. It is advisable to maintain the samples under vacuum at $\sim 0{ }^{\circ} \mathrm{C}$ so as not to impair their structural integrity.

In conjunction with this point, it is pertinent to note that a small amount of branched material, in contradistinction to the claims of others $[23,24]$, is formed during the polymerization of butadiene $[22,56]$. This appears to be due to the susceptibility of the vinyl group to attack by an organolithium. At high molecular weights $\left(\sim 10^{6}\right)$, gel has been observed [56] to form. This behavior is parallel to the branching and gelation encountered in the sodium- or potassiuminitiated polymerization of butadiene [78]. The formation of branched material is retarded by carrying out the polymerization at $0{ }^{\circ} \mathrm{C}$. At this temperature, at least several days are necessary for complete conversion.

The measurement of viscosity is a convenient mode of determining the molecular weight of polymers prepared by these homogeneous anionic polymerization techniques. Values for $K$ and $\alpha$ for several polymer-solvent pairs have been published; polystyrene [13,14], poly- $\alpha$-methylstyrene [17], polyisoprene $[20$,$] and polybutadiene [23,24].$

The retention of chain end activity of these polyorganolithiums has many important ramifications from the standpoint of opportunities to synthesize polymers 
of novel architecture, i.e., branched macromolecules. Morton [79] and Wenger [80,81] have synthesized tri- and tetra-functional "star" macromolecules from linear monodisperse polystyrene joined at one end through a common junction. Similar star species were created from polybutadiene [82]. Interesting viscosity studies have been performed on bulk and dilute constellations of these composite polymers. Trichloromethylsilane [82], silicon tetrachloride [79,82], 1,2,4-tri and 1,2,4,5-tetrachloromethylbenzene $[14,80,81]$ have been utilized as coupling agents. ${ }^{3}$ Branched polymers of the "comb" type have also been synthesized by coupling polystyryllithium to a partially chloromethylated polystyrene backbone [81] and by reacting acrylonitrile with poly-p-lithostyrene [83]. Yen [15] has used a tetrahydrofuran solution of $\alpha$ phenylethylpotassium to prepare virtually monodisperse polystyrene and star species. Utilization of the polystyrylpotassium carbanion results in a more efficient preparation of a star macromolecule, ascribable, in part, to the absence of any metal-halogen interconversion. Szwarc [84] has also outlined several ingeneous procedures by which star- and combshaped polymers might be synthesized.

An additional consequence of these systems is that linear block copolymers can be prepared by the selected addition of monomer according to a preconceived plan. After the complete conversion of monomer A, monomer $\mathrm{B}$ is added to the solution and polymerization again ensues. In this fashion, precise control is afforded over the number, sequence, and length of the blocks. Polymers can thus be produced having blocks of elastomeric and nonelastomeric segments.

An example of this type of polymerization is the preparation of a polystyrene-polyisoprene-polystyrene block copolymer. A polymer with good elastomeric properties is obtained when the segment molecular weights are about 10,000-50,000-10,000. These elastomers exhibit the high gum tensile strength of at least 3500 psi at an elongation of 1100 percent. The elastomeric properties of this composite macromolecule arise from the agglomeration of the glassy polystyrene segments. Hence, these aggregates act as cross-links.

Since participation in the network by a chain is dependent upon the diene unit having polystyrene segments at each end, it is important that the initiation reaction be accomplished as rapidly as possible. It is particularly important that no butyllithium be present at the cessation of the first styrene polymerization. The residual butyllithium would eventually react with isoprene and subsequently form an elastomerically ineffective chain. The slow initiation step is eliminated by the addition of a tertriary amine or anisole in amounts equal to the concentration of butyllithium. Initiation is thus completed in several minutes. The presence of either of these two components does not alter, to any detectable degree, the diene microstructure [85].

${ }^{3}$ The formation of star polymers with more than four chains per molecule has been reported. T. Altures, Jr., D. P. Wyman, V. R. Allen, and K. Meyersen, J. Polymer Sci 3A, 4131 (1965); J. Gervasi and A. B. Gosnell, J. Polymer Sci. 4A-1, 1391 (1966).
After depletion of the styrene, the desired amount of isoprene is added and polymerization again ensues. In hydrocarbon media, the rate of reaction of isoprene with a styryllithium polycarbanion is rapid [86]. Hence no problem similar to the slow $n$-butyllithiummonomer reaction is encountered. Following the complete incorporation of the diene, the desired amount of styrene is then added. Since the reaction of a dienyllithium with styrene is a relatively slow process, the addition of a trace amount of tetrahydrofuran is desirable to expedite the crossover step. In this fashion it is possible to prepare a copolymer with virtually monodisperse segments of any degree of polymerization. Butadiene can, of course, be used in lieu of isoprene. Copolymers with polybutadiene as the interior segment have achieved commercial importance as thermoplastic elastomers.

Table 1 lists some block copolymers. Many of these copolymers were prepared so as to compare their dynamic mechanical properties and thermal stability with those of random copolymers and mechanical blends of similar composition.

The selective termination of these polycarbanions permits the introduction of specialized functional groups at the ends of the chains. When the polymers in question possess two active sites, it is possible to form bifunctional macromolecules which may then be applied to further synthetic work. The addition of carbon dioxide to a solution of polycarbanions generates, after hydrolysis, chains with carboxylic endgroups [97-100]. Carboxyl terminated polystyrene is obtained by reacting the carbanion ends with succinic or phthalic anhydride [101]. Reaction with ethylene oxide introduces hydroxylic endgroups $[93,99,102]$. The introduction of terminal double bonds is effected by the addition of allyl chloride or allyl bromide to an ether solution of polystyrylsodium [101]. Terminal primary amine endgroups can also be added. This procedure consists of reacting $p$-aminoethyl benzoate with polystyrylsodium carbanions [103]. Michlers ketone also reacts quantitatively with polystyrylsodium carbanions [104-105].

These polymeric species have been utilized in a variety of ways. For example, polybutadiene with two hydroxylic groups may be reacted with an appropriate coupling agent, e.g., a trifunctional isocyanate, to yield an elastomeric network. This procedure unveils possibilities for novel applications in casting and molding polymers. Solid rocket fuel binders are one example of cast elastomers.

Cyclohexadiene [106] may, under the appropriate conditions, be polymerized to yield monodisperse rubber. The polymerization of methyl methacrylate by organolithium compounds [107] is kinetically complex and yields polydisperse polymer. Much of this complexity is the result of reaction of the organolithium reagents with the carbonyl group. Monodisperse poly(methyl methacrylate) has been prepared in tetrahydrofuran at $-75{ }^{\circ} \mathrm{C}$ by sodium biphenyl initiation [108]. The susceptibility of this monomer to electron transfer initiation was described earlier $[109,110]$. 
TABLE 1. Summary of block copolymers prepared in anionic polymerization

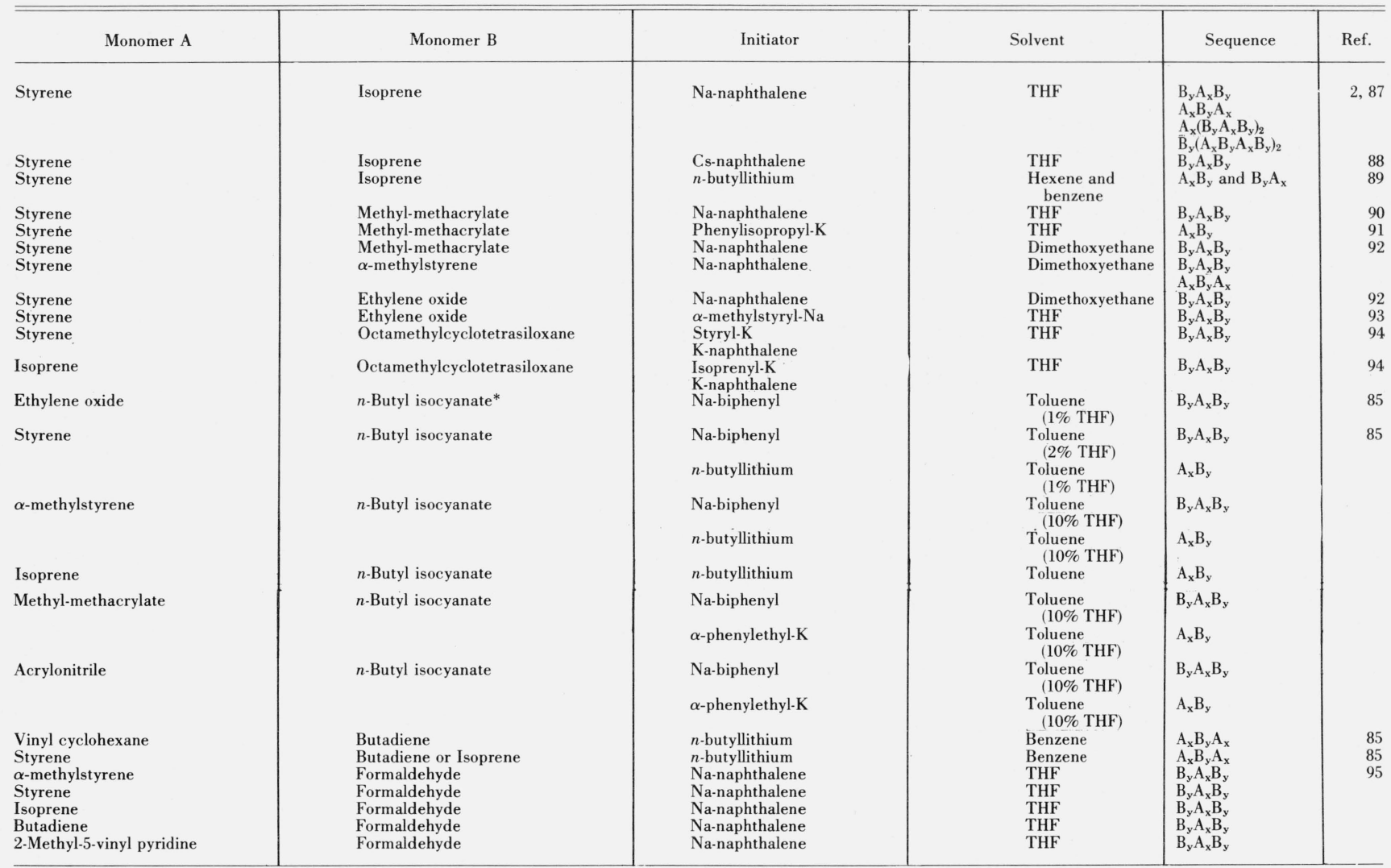

In the toulene-THF mixtures the amount of THF is expressed in volume percent.

In the toulene-THF mixtures the amount of THF is expressed in vol
*Poly-( $n$-butyl)isocyanate has been characterized as a rigid rod [96].

The presence of transfer reactions prohibits the preparation of monodisperse polymer from acrylonitrile or methacrylonitrile [111-114].

The guidance in years past of Dr. E. E. Bostick is gratefully recalled.

\section{References}

[1] M. Szwarc, Nature 178, 1168 (1956).

[2] M. Szwarc, M. Levy, and R. Milkovich, J. Am. Chem. Soc. 78 , $2656(1956)$

[3] P. J. Flory, J. Am. Chem. Soc. 62, 1561 (1940).

[4] R. Waack, A. Rembaum, J. D. Coombes, and M. Szwarc, J. Am. Chem. Soc 79, 2026 (1957)

[5] H. McCormick, J. Polymer Sci. 36, 341 (1959).

[6] F. Ẇenger, Makromoleculare Chem. 36, 200 (1960); 64, 151 (1963).

[7] M. Morton, R. Milkovich, D. McIntyre, and L. J. Bradley, J. Polymer Sci. 1A, 443 (1963).

[8] M. Morton, A. A. Rembaum, and J. L. Hall, J. Polymer Sci. 1A, 461 (1963).

[9] T. Lyssy, Helv. Chim. Acta. 42, 2245 (1959).

[10] H.-J. Cantow, Makromoleculare Chem. 30, 169 (1959).

[11] G. Meyerhoff, Z. physik Chem. (Frankfurt) 23, 100 (1960).

[12] F. M. Bower and H. W. McCormick, J. Polymer Sci. 1A, 1749 (1963).

[13] J. M. G. Cowie, D. J. Worsfold, and S. Bywater, Trans. Faraday Soc. 57, 705 (1961).
[14] T. Altares, D. P. Wyman and V. R. Allen, J. Polymer Sci. 2A, 4533 (1964).

[15] S.-P. S. Yen, Makromoleculare Chem. 81, 152 (1965).

[16] H. McCormick, J. Polymer Sci. 41, 327 (1959); F. Wenger J. Am. Chem. Soc. 82, 4281 (1960); Makromoleculare Chem. 37, $143(1960)$.

[17] A. F. Sirianni, D. J. Worsfold, and S. Bywater, Trans. Faraday Soc. 55, 2124 (1959).

[18] M. Morton, E. E. Bostick, and R. G. Clarke, J. Polymer Sci. 1A, 475 (1963)

[19] N. Calderon and K. W. Scott, J. Polymer Sci. 3A, 551 (1965).

[20] W. H. Beattie and C. Booth, J. Appl. Polymer Sci. 7, 507 (1963).

[21] S. E. Bresler, A. A. Korotkov, M. I. Mosevitsky, and I. Ya. Poddubnyi, Zhur. Tech. Fiz. 28, 114 (1958).

[22] J. F. Meier, Ph.D. Dissertation, University of Akron, 1963.

[23] H. Hsieh, J. Polymer Sci. 3A, 191 (1965).

[24] J. T. Gruver and G. Kraus, J. Polymer Sci. 2A, 797 (1964); 3A, 105 (1965).

[25] M. Morton and E. E. Bostick, J. Polymer Sci. 2A, 523 (1964).

[26] W. T. Grubb and R. C. Osthoff, J. Am. Chem. Soc. 77, 1405 (1955).

[27] C. Geacintov, J. Smid, and M. Szwarc, J. Am. Chem. Soc. 84, 2508 (1962).

[28] D. N. Bhattacharyya, C. L. Lee, J. Smid, and M. Szwarc, Polymer 5, 54 (1964).

[29] M. Szwarc and M. Litt, J. Phys. Chem. 62, 568 (1958).

[30] D. J. Worsfold and S. Bywater, Can. J. Chem. 38, 1891 (1960).

[31] B. D. Coleman, F. Gornick, and G. Weiss, J. Chem. Phys. 39, 3233 (1963).

[32] B. J. Mair, D. J. Termini, C. B. Willingham, and F. D. Rossini, J. Res. NBS 37, 229 (1946) RP1744.

[33] N. D. Scott and J. F. Walker, Ind. Eng. Chem. 32, 312 (1940).

[34] L. A. Wall, L. J. Fetters and S. Straus, Polymer Preprints 6 , 930 (1965). 
[35] R. K. Graham, D. L. Dunkelburger and W. E. Goode, J. Am. Chem. Soc. 82, 400 (1960).

[36] H. Gilman and A. H. Haubein, J. Am. Chem. Soc. 66, 1515 (1944).

[37] H. Gilman and F. K. Cartledge, J. Organometallic Chem. 2, 447 (1964).

[38] T. V. Talalaeva and K. A. Kocheskov, Zhur. Obshchei Khim. 23, 392 (1953); J. Gen. Chem. U.S.S.R. (Engl. trans.) 23, 399 (1953).

[39] H. Gilman and R. D. Gorsich, J. Org. Chem. 23, 550 (1958); A. G. Evans and N. H. Rees, J. Chem. Soc. 6039 (1963).

[40] M. Morton, E. E. Bostick, R. A. Livigni and L. J. Fetters, J. Polymer Sci. 1A, 1735 (1963).

[41] H. Hsieh, J. Polymer Sci. 3A, 163 (1965).

[42] G. L. Taylor, Ph.D. Dissertation, University of Akron, 1964.

[43] J. L. Hall, Ph.D. Dissertation, University of Akron, 1962.

[44] R. C. P. Cubbon and D. Margerison, Proc. Roy. Soc. 268A, $260(1962)$

[45] G. C. East, P. F. Lynch, and D. Margerison, Polymer 4, 139 (1963).

[46] I. Kuntz, J. Polymer Sci. 2A, 2827 (1964).

[47] D. J. Worsfold and S. Bywater, Can. J. Chem. 42, 2884 (1964).

[48] H. Hsieh, J. Polymer Sci. 3A, 153, 173 (1965).

[49] L. Gold, J. Chem. Phys. 28, 91 (1957).

[50] E. Bauer and M. Magat, J. Chim. Phys. 47, 841 (1950).

[51] D. Margerison and J. P. Newport, Trans. Faraday Soc. 59, 2058 (1963).

[52] L. J. Fetters, Ph.D. Dissertation, University of Akron, 1962.

[53] L. J. Fetters, J. Res. NBS 69A (Phys. and Chem.) No. 2, 159 (1965).

[54] B. Francois, V. Sinn, and J. Parrod, J. Polymer Sci. 4C, 375 (1964).

[55] H. Sinn, C. Lundborg, and O. T. Onsager, Makromoleculare Chem. 70, 222 (1964).

[56] M. Morton and L. J. Fetters, J. Polymer Sci. 2A, 3311 (1964).

[57] M. Morton, L. J. Fetters, and E. E. Bostick, J. Polymer Sci. 1 C, 311 (1963).

[58] S. Gadkary, Ph.D. Dissertation, University of Akron, 1963.

[59] G. Spach, M. Levy, and M. Szwarc, J. Chem. Soc. 355 (1962); M. Levy, M. Szwarc, S. Bywater, and D. J. Worsfold, Polymer 1, 515 (1960).

[60] M. A. Weiner, G. Vogel, and R. West, Inorganic Chem. 1, 654 (1962)

[61] S. Bywater and D. J. Worsfold, Can. J. Chem. 40, 1564 (1962).

[62] L. J. Fetters, L. A. Wall, and S. Straus, unpublished results.

[63] A. A. Rembaum, S.-P. S. Yen, and N. Indictor, J. Polymer Sci. 56, S17 (1962).

[64] L. J. Fetters, J. Polymer Sci. 2B, 425 (1964).

[65] F. A. Bovey and G. V. D. Tiers, Advances in Polymer Sci. 3, 139 (1963).

[66] R. J. Kern, Nature 187, 410 (1960).

[67] D. Braun, W. Betz, and W. Kern, Makromoleculare Chem. 42, 89 (1960).

[68] R. J. Kern, Polymer Preprints 4, No. 2, 324 (1963).

[69] D. J. Worsfold and S. Bywater, Makromolekulare Chem. 65, 245 (1963)

[70] D. M. Wiles and S. Bywater, J. Phys. Chem. 68, 1983 (1964).

[71] T. L. Brown, J. A. Ladd, and G. N. Newman, J. Organometallic Chem. 3, 1 (1965).

[72] H. McCormick, J. Polymer Sci. 25, 488 (1957).

[73] D. J. Worsfold and S. Bywater, J. Polymer Sci. 26, 299 (1957).

[74] D. J. Worsfold and S. Bywater, Can. J. Chem. 36, 1141 (1958).

[75] R. S. Stearns and L. E. Forman, J. Polymer Sci. 41, 381 (1959).

[76] I. Kuntz and A. Gerber, J. Polymer Sci. 42, 299 (1960).
[77] H. Hsieh, J. Polymer Sci. 3A, 181 (1965).

[78] I. Ya. Poddubnyi and E. G. Erenburg, Vysokomol. Soed. 2, 1625 (1960): Rubber Chem. Tech. 34, 975 (1961).

[79] M. Morton, T. E. Helminiak, S. D. Gadkary, and F. Bueche, J. Polymer Sci. 5 7, 471 (1962).

[80] T. A. Orofino and F. Wenger, J. Phys. Chem. 67, 566 (1963).

[81] F. Wenger and S.P. S. Yen, Polymer Preprints 3, [1] 162 (1962).

[82] R. P. Zelinski and C. F. Wofford, J. Polymer Sci. 3A, 93 (1965)

[83] M. B. Huglin. Polymer 5, 135 (1964).

[84] M. Szwarc, Makromoleculare Chem. 35, 132 (1960).

[85] L. J. Fetters, unpublished results.

[86] M. Morton, chap. VII in G. E. Ham, ed., Copolymerization (Interscience Oublishers. New York N.Y., 1964): S. Bywater, Advances in Polymer Sci. 4, 66 (1965).

[87] S. Schlick and M. Levy, J. Phys. Chem. 64, 883 (1960).

[88] A. Rembaum, F. R. Ells, R. C. Morrow, and A. V. Tobolsky. J. Polymer Sci. 61, 155 (1962).

[89] A. A. Korotkov, L. A. Shibayov, L. M. Pyrakov, V. G. Aldo shin, and S. Ya. Fenkel, Vysokomol. Soed. 1, 443 (1959).

[90] R. K. Graham, D. L. Dunkelberger, and E. S. Cohn, J. Poly mer Sci. 42, 501 (1960).

[91] D. Freyss, P. Remp, and H. Benoit, J. Polymer Sci. 2B, 217

[92] M. Baer, J. Polymer Sci. 2A, 417 (1964).

[93] D. H. Richards and M. Szware, Trans. Faraday Soc. 55, 1644 (1959).

[94] M. Morton. A. A. Rembaum, and E. E. Bostick, J. Appl. Polymer Sci. 8, 2707 (1964).

[95] K. Nore. H. Kawazura. T. Moriyama, and S. Yoshika, Makromolekulare Chem. 83, 35 (1965).

[96] H. Yu, A. J. Bur. and L. J. Fetters, J. Chem. Phys. 44, 2568 (1966); L. J. Fetters and H. Yu, Polymer Preprints 7, 443 (1966).

[97] C. A. Uranek, H. L. Hsieh, and O. G. Buck, J. Polymer Sci. 46, 535 (1960).

[98] H. Brody, D. H. Richards, and M. Szwarc, Chem. Ind. (London) 45, 1473 (1958).

[99] E. Schoenberg, J. Polymer Sci. 49, S9 (1961).

[100] D. P. Wyman, V. R. Allen, and T. Altares, Jr., J. Polymer Sci. 2A, 4545 (1964).

[101] P. Rempp and M. H. Loucheux, Bull. Soc. Chim. 1497 (1958).

[102] E. J. Goldberg, U.S. Patent 3,955,952 Sept. 25, 1962: Chem. Abstr. 58, 645f (1963).

[103] M. H. Loucheux, G. Mayer, and P. Rempp, Compt. Rend. 252, 2552 (1961).

[104] J. Trotman and M. Szware, Makromolekulare Chem. 37, 39 (1960).

[105] D. Braun and W. Fischer, Makromolekulare Chem. 85, 155 (1965).

[106] F. Dawans, J. Polymer Sci. 2A, 3297 (1964).

[107] D. M Wiles and S. Bywater, Trans. Faraday Soc. 61, 150 (1965).

[108] A. Rogi, J. E. Figueruelo, and E. Liano, J. Polymer Sci. 3B, 171 (1965).

[109] A. V. Tobolsky and D. B. Hartley, J. Am. Chem. Soc. 84, 1391 (1962).

[110] M. Szware and A. Rembaum, J. Polymer Sci. 22, 189 (1956).

[111] A. Tsukamoto, J. Polymer Sci. 3A, 2767 (1965).

[112] A. Zilkha, B.-A. Feit, and M. Frankel, J. Polymer Sci. 49, 231 (1961).

[113] C. G. Overberger, E. M. Pearce, and N. Mayes, J. Polymer Sci. 34, 109 (1959)

[114] P. Rempp, Polymer Preprints 7, 141 (1966).

(Paper 70A5-414) 